\title{
ZUM PROBLEM DES WESTLICHEN VORBILDS IN DER NEUEREN DISKUSSION ZUR POLITISCHEN ENTWICKLUNG*
}

\author{
Von Manfred Mols
}

In diesem Beitrag möchte ich einige Gedanken zum Problem und zur Chance des europäisch-nordamerikanischen Entwicklungsvorbilds aussprechen, und zwar aus der Sicht eines mit Entwicklungsproblemen befaßten Politikwissenschaftlers, dem es um die Schnitte zwischen Rationalität und Fragwürdigkeit bei der eigenen wissenschaftlichen Arbeit zu tun ist. Obwohl ich überwiegend konzeptuelle UUberlegungen anstelle, werde ich nicht immer - dies liegt in der Natur des politikwissenschaftlichen Geschäfts - der Versuchung zu praktisch-politischen Hinweisen widerstehen können.

Allgemein fällt an der neueren Entwicklungsdiskussion auf, wie sehr der nördlichatlantische Raum zwischen San Francisco und Moskau das in der Regel vorherrschende Leitbild für Entwicklung abgibt ${ }^{1}$. Selbst der relativ originellste Beitrag der Dritten Welt, die lateinamerikanische dependencia-Diskussion ${ }^{2}$, ist ja im Kern nichts anderes als das Aufgreifen der neueren marxistischen, „westlichen“ Imperialismus-Betrachtung aus der Sicht Betroffener in den sogenannten Peripherieländern. Die zentrale Frage bei all dem ist aber: Wieweit kann der „Westen“ überhaupt sinnvollerweise als Vorbild dienen und wo verstellt sein Gewicht nicht letztlich den Blick für adäquatere (?) Entwicklungsvorstellungen, die dann wohl besser „an Ort und Stelle" erarbeitet werden sollten, nämlich in der Dritten (und Vierten $\left.^{3}\right)$ Welt? Anders gewandt: Wieweit lassen sich Elemente im westlichen Paradigma aufzeigen, an die man außerhalb der Ersten und Zweiten Welt ${ }^{4}$ konstruktiv und ohne Imitationszwänge anknüpfen könnte, und was sollte man umgekehrt besser vermeiden?

\footnotetext{
* Das Folgende ist die überarbeitete und leicht erweiterte Fassung eines Vortrags, den ich auf Einladung von Prof. Hans-Werner Tobler im Mai vergangenen Jahres an der ETH Zürich gehalten habe. Ihm, den Mitgliedern des Interdisziplinären Nachdiplomkurses über Probleme der Entwicklungsländer sowie den Herren Prof. Peter Waldmann, Dipl.-Soz. Alfred Erlebach, Andreas Thimm, M. A. und Dr. Hans-Jürgen Puhle sei an dieser Stelle herzlich für kritische Anregungen gedankt.

1 Wichtige Ưbersichten über den Literaturstand bieten: Robert A. Packenham, Approaches to the Study of Political Development, in: World Politics 17 1964, $108 \mathrm{ff}$; Franz Nuscheler, Theorien zur politischen Entwicklung, in: Civitas, Bd. 8, 1969, $67 \mathrm{ff}$;; Fred W. Riggs, The Theory of Political Development, in: James C. Charlesworth (ed.), Ćontemporary Political Analysis, New York and London 1967, 317 ff.; Franz-Wilhelm Heimer, Begriffe und Theorien der politischen Entwicklung ${ }^{\alpha}$. Bilanz einer Diskussion und Versuch einer Ortsbestimmung G. A. Almonds, in: Dieter Oberndörfer (Hg.), Systemtheorie, Systemanalyse und Entwicklungsländerforschung, Berlin 1971, $449 \mathrm{ff}$; Michael Bohnet, Die Entwicklungstheorien - Ein Uberblick, in: ders. (Hg.), Das Nord-Süd-Problem. Konflikte zwischen Industrie- und Entwicklungsländern, München, 2. Aufl. 1971; Dirk Berg-Schlosser (Hg.), Die politischen Probleme der Dritten Welt, Hamburg 1972; Dieter Nohlen und Franz Nuscheler (Hgg.), Handbuch der Dritten Welt, Bd. I: Theorien und Indikatoren von Unterentwicklung und Entwicklung, Hamburg 1974.

2 Vgl. die Beiträge von Theotonio dos Santos, Osvaldo Sunkel, Celso Furtado, Fernando Henrique Cardoso, Rodolfo Stavenhagen und Aníbal Quijano bei Dieter Senghaas (Hg.), Imperialismus und strukturelle Gewalt. Analysen über abhängige Reproduktion, Frankfurt 1972; ders. (Hg.), Peripherer Kapitalismus. Analysen über Abhängigkeit und Unterentwicklung, Frankfurt 1974 (in beiden Bänden finden sich ausführliche Bibliographien zur dependencia-Problematik); vgl. auch den Ubersichtsartikel von Tilmann Tönnies Evers und Peter von Wogau, „dependencia ${ }^{\alpha}$ : lateinamerikanische Beiträge zur Theorie der Unterentwicklung, in: Das Argument 79, $1973,404 \mathrm{ff}$.

3 Es ist in letzter Zeit ubblich geworden - nicht zuletzt unter dem Eindruck der weltweiten Rohstoff situation -, die Armsten der Armen als Vierte Welt zu bezeichnen. Diesem Sprachgebrauch folge ich im obigen Text deshalb nicht, weil die Unterscheidung für die von mir angestellten Úberlegungen keine Bedeutung hat.

$4 \mathrm{Zu}$ dieser ${ }^{2}$ Welten ${ }^{\alpha}$-Einteilung vgl. Irving Louis Horowitz, Three Worlds of Development. The Theory and Practice of International Stratification, New York 1966.
} 
Diese Problemstellung ist alles andere als inaktuell. Zwar hat es in den letzten Jahren immer wieder Kritik am westlichen Vorbild, so wie es etwa in den "Modernisierungstheorien" zum Ausdruck kommt, gegeben ${ }^{5}$, doch bauen die geäußerten Vorbehalte und Einwände noch keine überzeugende nicht-westliche Alternative auf. Und vor allem: Wie dringend notwendig auch die verstärkte Mitdiskussion gerade der Dritten Welt über die Horizonte ihrer eigenen Zukunft sein wird und wie verdienstvoll und einflußreich nicht zuletzt die bisherigen Entwicklungsvorstellungen Internationaler Organisationen gewesen sein mögen, da sie, wie Dieter Nohlen und Franz Nuscheler ${ }^{6}$ jüngst bemerkten, sich „um eine universell anerkannte Wert- und Prioritätensetzung bemühen müssen, die noch am ehesten dem Vorwurf entgegenwirken kann, daß sich in diesem Entwicklungsbegriff lediglich die Wert- und Zielskala der eigenen Gesellschaft oder eigenen gesellschaftlichen Leitbilder reproduzieren" - vorläufig bleibt die manifeste wie latente Attraktion des Westens bestehen; denn der gravierende Entwicklungsvorsprung der Ersten und Zweiten Welt kann nicht gut übersehen oder gar bestritten werden. Und vorläufig bleibt auch das Legat der westlichen Erfahrung und das Problem seiner Vermittlung durch uns, die westlichen Sozialwissenschaftler.

Es wäre reizvoll gewesen, stärker, als dies im folgenden Text möglich sein wird, in der Dritten Welt überlegte Zielvorstellungen ${ }^{7} \mathrm{zu}$ berücksichtigen. Obwohl solche Gedankengänge in der Tat gelegentlich aufgegriffen werden, steht das vorhin formulierte Anliegen an erster Stelle. Die Argumentation wird sich dabei in zwei Schritten vollziehen: Wenn man über politische Entwicklung räsoniert, hebt man bekanntlich ab auf eine Besonderheit innerhalb der breiter angelegten neueren Entwicklungsdiskussion. Folglich wird es erstens um die Begriffe „Entwicklung“ und "Unterentwicklung" in einem allgemeineren Sinne und dann in einem zweiten Abschnitt um den Stellenwert von politischer Entwicklung innerhalb der breiter geführten Entwicklungsdiskussion gehen.

\section{Zur zeitgenössischen Diskussion von Entwicklung - Unterentwicklung}

Mit dem Konzeptpaar Entwicklung - Unterentwicklung ${ }^{8}$ meint man das Niveaudifferential von Kulturen in geographisch und historisch fixierbaren Räumen - Kulturen hier verstanden als komplexe und tendenziell in sich stimmige Systeme aus gesellschaftsspezifischer Rationalität, Wirtschaftsformen, politischen Strukturen, Verhaltensweisen, Zukunftserwartungen, Außenorientierungen ... Kulturelle Niveaugefälle gehörten immer zur menschlichen Geschichte. Neu für die gegenwärtige historische Epoche, welche mit der europäischen Aufklärung anhebt und in der zeitgenössischen Entwicklungsdiskussion einen ihrer kennzeichnendsten Ausdrücke findet, sind:

5 Vgl. Franz Nuscheler, Bankrott der Modernisierungstheorien?, in: Nohlen/Nuscheler (zit. Anm. 1), 195 ff. 6 Nohlen/Nuscheler (zit. Anm. 1) 17

7 Vgl. außer den Beiträgen aus der Dritten Welt bei Berg-Schlosser und Bohnet (beide zit. Anm. 1): Charles W. Anderson, Fred R. von der Mehden, Crawford Young, Issues of Political Development, Englewood Cliffs, 2. Aufl. 1974.

8 Die unmittelbar folgenden Passagen schließen sich an an: Manfred Mols, Parteien und Entwicklung in der Dritten Welt. Theoretisch-methodologische Uberlegungen; illustriert am Beispiel des mexikanischen Partido Revolucionario Institucional, in: Wolfgang Jäger ( $\mathrm{Hg}$.$) , Partei und System. Eine kritische Ein-$ führung in die Parteienforschung, Stuttgart u. a. 1973, 210 ff., hier bes. 211-213. 
- Die Vorstellung, daß sich am Faden einer "gradlinig verlaufenden Zeit" (Dreitzel) Fortschritt als qualitative Verbesserung menschlicher Existenzbedingungen akkumuliert;

- das Selbstvertrauen, den wichtigsten Faktoren dieses Fortschritts durch die Anstrengung des menschlichen Geistes auf die Spur zu kommen;

- hierbei Universalien zu entdecken, die, philosophisch und anthropologisch legitimiert durch das Postulat der einen Menschheit, als Bedingungen zur Verbesserung der menschlichen Lebensqualität für alle Völker und Regionen gelten werden;

- die Selbstsicherheit, dies alles sozialtechnologisch in die Praxis umsetzen zu können: Fortschritt - so die allgemeine Ưberzeugung unserer Zeit (und bezeichnenderweise haben sich hier die Entwicklungstheoretiker nicht von den Werbeoffizieren einer postindustriellen Gesellschaft unterschieden ${ }^{9}$ ) - ist in gleicher Weise mach- wie interkulturell transferierbar.

Wie immer die Einzelheiten aussehen mögen: Insgesamt wird Unterentwicklung begriffen (oder ist zumindest überwiegend begriffen worden ${ }^{10}$ ) als ein Defizit an jenen Modernitäten, die im Saldo etwa das ausmachen, was man als die westliche Industriegesellschaft mit ihren politischen Kerninstituten zu beschreiben pflegt. Was dann umgekehrt oft dazu geführt hat, daß man nicht mehr zwischen „entwickelt", "modern" und „westlich" unterscheiden kann"1. Entwickelt ist also das, was uns im Westen als unsere Welt umgibt. Schon im bloßen Begriff Entwicklungsländer zahlen wir einen semantischen Vertrauensvorschuß auf ein Potential, unserem Ebenbild und Gleichnis nahe kommen zu können. Bei Lichte besehen mag dies alles eine merkwürdige Mischung aus Naivität und Selbstüberzogenheit sein, doch muß man zunächst darauf aufmerksam machen, daß hier nicht einmal die höheren Weihen der Geistesgeschichte fehlen. Grundsätzlich teilt die westliche Entwicklungsdiskussion - heute um den nordamerikanischen Anschauungs- und Problemhorizont erweitert - den eurozentrischen Fortschrittsglauben der Condorcet, Comte, Marx, Spencer, selbst Max Weber ... .2 ein Fortschrittsoptimismus, der in gleicher Weise vom Selbsterfahrungsprozeß der industriestaatlich-kapitalistischen Welt des Okzidents inspiriert ist wie vom neuzeitlich-philosophischen Evolutionsdenken. Wobei nur noch hinzuzufügen bliebe, daß auch die partielle Negation dieser Welt in den Prospekten des europäischen Sozialismus (wie vorhin mit dem Hinweis auf Marx angedeutet) immer nur Alternative im gleichen historischen Potential bleibt. Wenn etwa Irving Louis Horowitz ${ }^{\mathbf{1 3}}$ von drei entwicklungstypischen Welten im gegenwärtigen Zeitalter gesprochen hat, so sind die Erste und die Zweite Welt allenfalls die verschiedenen Seiten der nämlichen Münze. Die

9 Samuel P. Huntington (The Change to Change, Modernization, Development, and Politics, in: Comparative Politics 3, 1971, $283 \mathrm{ff}$., hier Anm. 17) weist in diesem Zusammenhang auf Daniel Bell und Zbigniew Brzezinski hin („Both stand generally in the optimistic stream . . .). Stellvertretend für einen recht großen Kreis dieser futurologischen Optimisten sei hier noch genannt: Robert Jungk, Der JahrtausendMensch. Bericht aus den Werkstätten der neuen Gesellschaft, München u. a. 1973. Auch der ${ }_{n}$ Bericht der ,Kommission für das Jahr 2000‘, Der Weg ins Jahr 2000, München u. a. 1968, liegt ganz auf der von Huntington charakterisierten Linie. Immerhin soll hier nicht unterschlagen werden, daß man seit einiger Zeit eine Gegenströmung feststellen kann, auch wenn es mir hier nicht möglich ist, dem im Detail nachzugehen.

10 "The bridge across the Great Dichotomy between modern and traditional societies is the Grand Process of Modernization. ${ }^{\circ}$ Huntington, The Change to Change and traditional societies is the Grand Process Endruweit, Der Schritt zur Industriegesellschaft. Der Begriff der Industriegesellschaft als Berührungspunkt zwischen Industriesoziologie und Entwicklungssoziologie, in: KZS XIX 1967, $454 \mathrm{ff}$.

11 Vgl. Huntington (zit. Anm. 9), 294.

12 Den oben im Text genannten Namen ließen sich leicht weitere an die Seite stellen: Darwin, Tönnies, Sir Henry Maine (als bekanntester früher Vertreter des amerikanischen soziologischen Fortschrittsoptimismus) ... Vgl. außer dem jetzt mehrfach zit. Huntington-Aufsatz: Hornell Hart, Social Theory and Social Change, in: Llewellyn Gross (ed.), Symposium on Sociological Theory, New York u. a. $1959,196 \mathrm{ff}$. 13 In der Anm. 4 zit. Arbeit. 
Dritte Welt ist dann etwas, dem man die ersten beiden Welten als die eigentlich empirisch sinnvollen Orientierungsmöglichkeiten vorgegeben hat. Das Ganze modifiziert, wenn es hochkommt, durch die eine oder die andere regional- und/oder länderspezifische Variation.

Wir sind jetzt an einem etwas schwierigen Punkt unserer Úberlegungen angelangt. Auf der einen Seite ist der universalhistorische Anspruch des westlichen Vorbilds gewiß bedenklich, auf der anderen Seite kommt man nicht um die Frage herum, ob es nicht doch für die Dritte Welt auswertbare Erfahrungen des westlich-industriestaatlichen Modells gibt.

Das Bedenkliche am westlichen Vorbild läßt sich noch am einfachsten andeuten. Es sind dies: seine historisch begrenzte Wiederholbarkeit und seine nicht unbedingt $\mathrm{zu}$ wünschende Reproduktion.

Was die historisch begrenzte Wiederholbarkeit angeht, so denke ich sowohl an den ungeheuren Zeitdruck für jede gegenwärtige Entwicklungspolitik ${ }^{14}$ als auch an solche, nicht nach Belieben raumzeitlich verfügbaren Entwicklungsfaktoren wie die großen und dann nachfolgende Gesellschafts- und Verfassungsformen prägenden Revolutionen der euroamerikanischen Neuzeit (etwa im englischen Amerika, in Frankreich, in Rußland); an die europäische Aufklärung; an die religionsgeschichtlichen Vorbedingungen des Kapitalismus mit ihren beiden wohl interessantesten Folgen: an ein nahezu selbstzweckliches Arbeits- und Berufsethos ${ }^{15}$ und an die innerweltliche Askese hinsichtlich der Konsumanteile der wirtschaftlichen Produktion (zwischen den diesbezüglichen Typisierungen Max Webers und den Idealen marxistischer Politökonomen scheint mir hier kein substantieller Unterschied zu bestehen); übrigens auch an die imperialistisch profitable Situation des westlichen Entwicklungsweges in den letzten 200 oder 300 Jahren ... Gewiß mag man sich hier über die Gewichtung von Einzelheiten streiten, aber es sind doch Einzelheiten, die die beiden folgenden Argumente, die problematische Wiederholbarkeit der westlichen Entwicklungssituation betreffend, verständlich machen sollten: Erstens ist die Moderne, eben die westliche Moderne, eine Erfahrungsgesamtheit, die sich nur in der wissenschaftlichen Analyse ex post factum als Summe identifizierbarer und überwundener Modernisierungsdefizite nachzeichnen läßt; denn realgeschichtlich hat sich das Ganze als ein allenthalben ineinandergreifender Ereigniskomplex vollzogen. Die mangelnde Reproduzierbarkeit des Westens liegt dann vor allem daran, daß jede umfassende (gleichsam alle relevanten Faktoren enthaltende) Durchführung von abermaliger analoger Entwicklung mit Sicherheit sowohl jedes entwicklungspolitisch vertretbare Zeitbudget als auch die planerisch-technologischen Fähigkeiten der beteiligten Akteure - seien dies nun Länder, Regierungen, dynamisierte soziale Straten und/oder Gruppen oder sonstige change agents - übersteigen würde. Zweitens kommt noch hinzu, daß es heute für die Dritte Welt - in entschiedenem Gegensatz zum europäisch-nordamerikanischen Werdengang des 17., 18. und 19. Jahrhunderts - weder eine referenzgruppenfreie noch vor allem eine von den politischen und wirtschaftlichen und ideologischen Bedürfnissen und Zwän-

14 Kostenhinweise für entwicklungspolitische Langsamkeiten finden sich u. a. in Mihailo Mesarovicl Eduard Pestel, Menschheit am Wendepunkt. 2. Bericht an den Club of Rome zur Weltlage, Stuttgart 1974; vgl. auch Erhard Eppler, Wenig Zeit für die Dritte Welt, Stuttgart/Berlin 1971.

$15 \mathrm{Da}$ gerade dies für die Dritte Welt alles andere als selbstverständlich ist, demonstriert René König, Uber einige offene. Fragen und ungelöste Probleme der Entwicklungsforschung, in: KZS, Sonderheft 13, Aspekte der Entwicklungssoziologie, 1969, 9 ff., hier bes. $20 \mathrm{ff}$. 
gen und Assistenzen der Ersten und Zweiten Welt unbetroffene Selbstfindung im Sinne eines großzügig bemessenen „trial and error" geben kann.

Was nun die nicht einmal unbedingt zu wünschende Reproduktion des Westens angeht, so möchte ich einfach an die allenthalben greifbaren Krisenerscheinungen unserer euroamerikanischen Gegenwart erinnern dürfen ${ }^{16}$. Nicht in allem überzeugend wäre das universalhistorisch verstandene westliche Entwicklungspostulat schon deshalb, weil sich angesichts der politischen, sozioökonomischen und kulturellen Defekte der spätindustriellen Gesellschaft ihr früher so unkritisch wie oft behaupteter Vorbildcharakter ausschließt. Um einen einzigen Hinweis zu geben: Eine Gesellschaft, die die Ausweitung der industriellen Produktion unhinterfragt zum Ausweis ihrer Fortschrittseinlösung gemacht hat, wird sich - hier würde ich alle Bedenken des Klubs von Rom voll unterschreiben - auf längere Zeit selbst zugrunde richten. Wollte man dieses and anderes - etwa das, was Marcuse und Touraine ${ }^{17}$ über Manipulation und die Macht der Apparate geschrieben haben der Dritten Welt uneingeschränkt zur Nachahmung anbieten, versuchten wir einen Export von Problemen, von denen wir nicht wissen, ob wir je mit ihnen fertig zu werden vermögen und die, falls weltweit imitiert, mit großer Wahrscheinlichkeit das Ende lebenswerter Menschlichkeit bedeuten würden.

Und die trotz allem auswertbaren Erfahrungen des westlich-industriestaatlichen Modells? Damit sind wir bei der Entwicklungsdiskussion in einem engeren, $d$. h. wissenschaftlich wie praktisch professionellen Sinne angelangt. Auf die soeben gestellte Frage möchte ich drei einander ergänzende Antworten geben.

Die erste Erfahrung heißt: Wir sind sensibilisiert worden für den Unterschied zwischen Modernisierung und Entwicklung. Modernisierung ist die Überwindung von konkreten Transformationsdefiziten mehr oder gänzlich traditionaler Gesellschaften im Hinblick auf Struktureigenschaften, Einstellungen und Kapazitätsniveaus in der modernen industriellen und spätindustriellen Gesellschaft. Geleistete Modernisierungen stellen noch keine Entwicklung her, aber sie gehören dazu und sind in manchem deren Voraussetzung. Modernisierungen können als Erfahrungen angesehen werden, auf die die heutige Dritte Welt schon aus Gründen der historischen Okonomie nicht verzichten kann und die daher konsequenterweise in den entwicklungspolitischen Problemhorizont der Eliten der Entwicklungsländer bzw. oft genug in das Bewußtsein veränderungsdynamischer Gegeneliten eingegangen sind. Zeit und Not drängen in der Dritten Welt zu sehr, als daß man sich exklusiv auf länger dauernde Experimente eines eigenen Weges für die Überwindung von Überbevölkerung und Massenarbeitslosigkeit, administrativer Korruption und schichtenspezifischen Distributionsverzerrungen einlassen könnte. Daniel Lerner ${ }^{18}$ schreibt daher zu Recht im Vorwort seiner Untersuchung über einige Länder des Nahen Ostens: „Wir werden ... zeigen, daß das westliche Modell der Modernisierung gewisse Komponenten und Sequenzen aufweist, die universell relevant sind.“ Oder in den Worten von Samuel Huntington ${ }^{19}$ : „Es ist möglich, verschie-

16 Vgl. statt vieler die Anm. 14 zit. Arbeit von Mesarović/Pestel, ferner: Dennis Meadows u. a., Die Grenzen des Wachstums. Bericht des Club of Rome zur Lage der Menschheit, Stuttgart 1972; Die Energiekrise. Episode oder Ende einer Ara?, Hamburg 1974 (Die Zeit-Bücher).

17 Herbert Marcuse, Der eindimensionale Mensch. Studien zur Ideologie der fortgeschrittenen Industriegesellschaft, Neuwied und Berlin 1970 (am Orig. zuerst 1964); Alain Touraine, Die postindustrielle Gesellschaft, Frankfurt 1972 (franz. Orig. 1969).

18 Daniel Lerner, Die Modernisierung des Lebensstils: eine Theorie, in: Wolfgang Zapf (Hg.), Theorien des sozialen Wandels, Köln/Berlin 1969, $362 \mathrm{ff}$., hier 362 (Es handelt sich um eine Ubersetzung aus: The Passing of Traditional Society, Glencoe/Ill. 1958).

19 Huntington (zit. Anm. 9), 289. 
dene Ebenen oder Phasen von Modernisierung zu unterscheiden, durch die alle Gesellschaften hindurch müssen." Denn man muß - um diese stellvertretend für eine ganze Generation von Entwicklungstheoretikern aufgegriffenen, wissenschaftlicher Beobachtung entwachsenen Ratschläge für eine praktische Entwicklungspolitik ${ }^{20}$ einmal frei zu konkretisieren - auch in den Ländern der Dritten Welt strukturelle Anreize zur sozialen Mobilität bilden, um jene Desintegrationstendenzen traditionaler Gesellschaften zu überwinden, die gesamtgesellschaftliche Arbeitsteilung kaum zulassen; man muß Formen gesellschaftlicher Rationalität erzeugen, die den Unternehmer und/oder den Planer ermöglichen; man muß die Stabilität struktureller Handlungsmuster ungleich dauerhafter als bisher üblich auch institutionell absichern; man muß - um es kurz zu machen - in vieler Hinsicht nachahmen, um überleben zu können. Und was für jede praktische Entwicklungspolitik besonders naheliegt: Auch die konzeptuell-theoretischen Erträge der westlichen Modernisierungsdiskussion haben zumindest durchgängig den Wert eines entwicklungspolitischen Kontrasts, einer topischen Konfiguration von Urteilen, eines "Steinbruchs". Aber nochmals: Unsere Sensibilisierung für den Unterschied zwischen Modernisierung und Entwicklung darf uns nicht vergessen lassen, daß Modernisierung allenfalls so etwas wie ein take-off für Entwicklung ist. Entwicklung oder besser: Entwickelt-Sein ist mehr, und zwar auch mehr als die Summe der Modernisierungsteile.

Die eigentümliche Qualität von Entwicklung ist die zweite Erfahrung aus dem westlich-industriestaatlichen Vorbild. Historisch haben sich die westlichen Nationen, die hier das Anschauungssubstrat abgeben, zur Moderne nach selbst vermittelten Bedürfnissen entwickelt, jedenfalls nicht durch den Antrieb eines Strukturgefälles von der Größenordnung, wie es heutzutage zwischen unterentwickelten und entwickelten Ländern besteht.

In den unterschiedlichsten Lagern der gegenwärtigen Entwicklungsdiskussion kommen die auswärtigen Referenzgruppen-Standards zum Tragen: David McClellands ${ }^{21}$ achievement-motivation-Beitrag, das Metropole-Satelliten-Schema der neueren Theorien des peripheren Kapitalismus ${ }^{22}$ und selbst noch die seinerzeit von Raúl Prebisch $^{23}$ vorgetragenen Strategien handelspolitischer Positionsverbesserungen haben einen gemeinsamen empirischen und schließlich auch analytischen Hauptnenner: die Anerkennung der extrem gewichtigen Dominanz externer Impulse bzw. einer Bezugswelt, an deren Erschaffung die Entwicklungsländer nicht oder allenfalls passiv beteiligt waren.

Im Gegensatz dazu muß man den Werdegang der heute als entwickelt geltenden Länder anders beurteilen. Bekanntlich war Entwicklung auch hier nie rein endogen. Auch hier also bildeten Akkulturationsanreize im weitesten Sinne immer wieder den stimulierenden Motor. Nur überwiegt insgesamt in den Akkulturationsbilanzen ein wechselseitiges Geben und Nehmen. Und was hier noch wichtiger ist: Die partiellen Innovationsvorsprünge des Nachbarn konnten in der Regel von den nachziehend rezipierenden Völkern durchaus aufgearbeitet oder bedürfnisspezifisch modifiziert werden. Funktionale Gewaltenteilung etwa und eine allgemeine Gerichtsbarkeit, ein allgemeines Bildungswesen und die sozialstaatliche Sicherung

20 Auf die schon um 1960 sehr deutlichen praktischen Entwicklungsabsichten der „political-development-“ Theoretiker geht Heimer ein (zit. Anm. 1), $450 \mathrm{f}$. (dort ausführliche Literaturhinweise).

21 David C. McClelland, The Achieving Society, Princeton 1961

$22 \mathrm{Vgl}$. die ausgezeichnete Einführung von Dieter Senghaas, Elemente einer Theorie des peripheren Kapitalismus, in: ders., Peripherer Kapitalismus ... (zit. Anm. 2).

23 Nachweise bei Bohnet (zit. Anm. 1), 301; vgl. auch den bei Bohnet (115 ff.) abgedruckten Beitrag von

Prebisch, Die Rohstoffexporte und die Verschlechterung der terms of trade. 
der individuellen Existenz sind ein gesamteuropäisches Erbgut geworden, auch wenn die konkrete Ausgestaltung solcher „westlicher Universalien“ den Interpretationsmaximen der unterschiedlichen Herrschaftssysteme unterliegt.

Ich hatte vorhin Wert gelegt auf die Feststellung eines Unterschiedes zwischen Modernisierung und Entwicklung. Viele Entwicklungsländer haben längst die europäischen Pro-Kopf-Einkommen der 50er Jahre erreicht oder überschritten, erstaunliche Industrialisierungsniveaus durchgesetzt und vereinzelt sogar verfassungsstaatliche Rationalisierungsformen des politischen Prozesses eingeübt. Dennoch sind die gleichen Länder weder dem Status der Unterentwicklung entkommen noch sieht es so aus - und dies scheint mir selbst für über Nacht reich gewordene OPEC-Länder zu gelten -, daß dies in absehbarer Zeit geschehen würde. Und zwar deshalb nicht, weil sie noch nicht ihre Bezugswelt und ihre eigenen Lebensqualitäten jenseits eines westlichen Instrumentariums (oder Zieles?) aktiv mitzusetzen wußten und weil für die meisten von ihnen auch keine Aussicht besteht, dem eigentlichen Kainszeichen der Unterentwicklung, der einseitigen Adaptation, zu entsagen. Ich halte daher fest: Die Strukturgefälle zwischen Entwicklung und Unterentwicklung zeichnen sich nicht nur durch die Menge der von Fall zu Fall gegebenen Modernisierungsdefizite aus - die meisten davon lassen sich durch den Einsatz technischer und sozialtechnischer Mittel nach und nach überwinden - : Zur Entwicklung gehört auch und vor allem eine relative Zielsetzungsautonomie, d. h. eine durch Modernisierungsbestrebungen allenfalls eingeleitete Dynamik muß zur Eigenstabilität finden und damit übergeleitet werden in die Diskussion um eigene Ziele, in das Experimentieren mit eigenen Strukturen und schließlich in jenes vorhin idealisierte gleiche Austauschverhältnis eines wechselseitigen Gebens und Nehmens in zwischenstaatlichen und/oder regionalen Akkulturationen/Kooperationen/ auch Konflikten. In den Worten von Horowitz ${ }^{24}$ : „Die Dialektik der Entwicklung ist so beschaffen, daß die Transformation von Nachahmung in eine Erneuerung für die Dritte Welt essentiell ist."

Ich sollte hinzufügen, daß Überlegungen dieser Art immer mehr an Rang in den Ländern der Dritten Welt selbst gewinnen ${ }^{25}$ und daß man auch mit Hilfe systemtheoretischer und kybernetischer Ansätze zum gleichen Resultat kommt. Verwiesen sei hier etwa auf Fred Riggs'26 Ausführungen zur Ókologie sozialer Systeme oder auf die seit Jahren anhaltende Diskussion um systemische Problemlösungskapazitäten ${ }^{27}$. Auch Richard F. Behrendts ${ }^{28}$ im kultursoziologischen Vergleich gewonnener Begriff der Entwicklungsdynamik weist in diese Richtung. Damit hier kein Mißverständnis auftaucht: Relative Zielsetzungsautonomie hat nichts mit den zwischenstaatlich intendierten Autarkie-Überlegungen vergangener Jahrzehnte zu tun, sondern hebt $\mathrm{ab}$ auf eine leidlich autonom mitgesteuerte Umwelt durch das Vermögen eines sozialen Systems, mit seinen Nachbarsystemen (hier z. B.: Nachbarstaaten/Industriestaaten/Erste und Zweite Welt/auch: multinationale Konzerne u. dgl.) in einem actio-reactio-Gleichgewicht zu leben. Im übrigen stimme ich hier gern Donald McGranahan ${ }^{29}$ zu, wenn er anläßlich der Präsentation des sogenann-

\footnotetext{
24 Horowitz (zit. Anm. 4), 324.

25 Nachweise bei Mols (zit. Anm. 8), dort Anm. 18.

26 Fred W. Riggs, The Ecology of Development, Comparative Administration Group Occasional Paper, Bloomington 1964; Riggs wiederholt diese Uberlegungen in dem Anm. 1 zit. Aufsatz.

27 Vgl. Heimer (zit. Anm. 1), 470 f.; Nuscheler (zit. Anm. 5), 205.

28 Richard F. Behrendt, Soziale Strategie für Entwicklungsländer, Frankfurt 1965.

29 Donald McGranahan, Entwicklungsindikatoren und Entwicklungsmodelle, in: Nohlen/Nuscheler (zit. Anm. 1), $208 \mathrm{ff}$., hier 217.
} 
ten „Kapazitäts-Leistungs-Modells“ meint, daß diesbezügliche Definitionen mehr die Funktion besäßen, den Orientierungsrahmen der Forschung zu bestimmen als daß dies schon alles so präzise sei, daß man sich darauf in praktischer Entwicklungsabsicht einigen könne. Dies ist richtig - und dennoch: Was man in bezug auf Problemlösungskapazität und/oder relative Zielsetzungsautonomie an Kriterien postuliert, entspricht, wie systemtheoretisch abstrakt auch gewonnen, auf der anschaulicheren Stufe historisch lebensweltlicher Interpretation westlich neuzeitlicher Erfahrung.

Die dritte Erfahrung aus dem westlichen Vorbild muß etwas umständlicher abgeleitet werden. So problematisch es ist, jahrzehntelange wissenschaftliche Diskussionen auf eine charakterisierende Formel $\mathrm{zu}$ bringen, so sehr würde ich doch zu dem Urteil neigen, daß die allermeisten Arbeiten - wenn sie nicht überhaupt bloß irgendwelche Entwicklungstrends mit schnellfertig beschlossenen Indikatoren „belegen“ sollten - Modernisierungstheorien "mittlerer Reichweite“ brachten.

Man kann aus dieser sicher nicht ganz befriedigenden Situation verschiedene Konsequenzen ziehen. Man kann die Theorien vermehren in der Hoffnung, bisherige Hypothesen/Argumentationspunkte/Faktoren durch essentiellere zu ersetzen bzw. durch eine auf diese Weise vergrößerte „notwendige“ Pluralität von Beiträgen möglichst alle relevanten Entwicklungstopoi zu berücksichtigen. Wenngleich dies eine sicherlich recht praktische Forschungsstrategie ist, vermag sie im grundsätzlichen deshalb nicht recht zu überzeugen, weil man bei ihr entweder auf alle Fallstricke essentialistischer Aussagen tritt oder mit dem vorhin angedeuteten Prinzip in Konflikt gerät, daß das Ganze mehr ist als die Summe seiner Teile: Die Ausweitung des Partikularen wird nie über die partielle Modernisierungsdiskussion hinausführen. Man kann zweitens längerfristig - dies schwebt z. B. den Herausgebern des „Handbuchs der Dritten Welt"30" vor - eine "Synthese von bisher rivalisierenden Theoriestücken " anzustreben. Obwohl mir Nuschelers Plädoyer für eine „integrierte Theorie“ in den Intentionen einleuchtet, kann ich mich der beiden praktischen Eindrücke nicht erwehren, daß wir dabei schlicht an Theoriebildungsproblemen scheitern würden (Hypothesen mittlerer Abstraktheit wären probabilistisch zu formulieren. Bei Vorlage probabilistischer Sätze läßt sich aber keine zwingende Hierarchisierung mehr vornehmen, da das jeweilige explicandum keine notwendige Deduktion aus explicans + Randbedingungen ist ${ }^{31}$ ) oder, sollte man diese Theorien jenseits wissenschaftslogischer Verfahrungsgrundsätze ansiedeln wollen, allen reduktionistischen Zufälligkeiten jener Grand Theories ausgesetzt wären, die Merton ${ }^{32}$ seinerzeit in der Auseinandersetzung mit Parsons überzeugend geschmäht hat.

Mir scheinen angesichts dieses mehrfachen Dilemmas zwischen Forschungsidealen und Forschungspraxis die Modernisierungstheorien noch eine andere Konsequenz offen zu lassen - und das wäre die dritte Erfahrung: Man kann sich zu einer Art phänomenologischer Reduktion entschließen in der Weise, daß man in einer Rückbesinnung auf abstrakte und gemeinsame dynamische Entwicklungsimpulse jenseits der bisher erfahrenen historischen Zufälligkeiten nach Elementen sucht, mit deren Hilfe sich modell-gerichtete und/oder theoriefähige Überlegungen anstellen lassen, die zwar noch die Grunderfahrungen des Westens berücksichtigen, für sich

30 Nohlen/Nuscheler (zit. Anm. 1), hier bes. 204 in dem Anm. 5 zit. Beitrag von Nuscheler.

$31 \mathrm{Vgl}$. Manfred Mols, Der Anspruch generaltheoretischer Exaktheit: Bemerkungen zu einigen Arbeiten politikwissenschaftlicher Systemanalyse, in: Oberndörfer (zit. Anm. 1), 63 ff., hier 105.

32 Robert K. Merton, On Theoretical Sociology. Five Essays Old and New, New York and London 1967. 
selbst aber keine platten Imitationsvorschläge mehr bedeuten. Ein solches Vorgehen - das sich im Ansatz im Entwicklungssyndrom des Committee on Comparative Politics of the Social Science Research Councilis3 zeigt (und ich sage dies, ohne mich gleichzeitig hinter dessen interpretatorische Mischung aus Leviathanismus und Elitentheorie zu stellen ${ }^{34}$ ) - böte deutliche Vorteile: Man könnte die bisherigen und übrigens weiterhin uneriäßlichen Modernisierungstheorien mittlerer Reichweite stärker als bisher einander zuordnen und damit positv ihren relativen Aussagewert anerkennen, ohne sich vorzeitig um Substitutionen und Integrationen zu bemühen; aus ihrer auf diese Weise überschaubar gestalteten faktischen Komplexität ließen sich entwicklungspraktische Leitsätze gewinnen und in ihrer partiellen Wirksamkeit und Unwirksamkeit erkennen; man könnte Entwicklungsmuster auf der Basis historischer Wahrscheinlichkeiten diskutieren, ohne mit Entwicklungsmodellen aufzuwarten, die nichts als der reine Abklatsch bisheriger Exempel im Westen sind. Man würde - um es zu wiederholen - Grunderfahrungen im westlichen Vorbild zur Verfügung stellen, ohne sie gleich zur endgültigen Norm künftiger Entwicklungswege zu nehmen. Um ein einfaches Beispiel zu bringen: Entwickeltsein ist nach westlicher Grunderfahrung (das würde man in Frankfurt und Warschau, in Salt Lake City wie in Moskau nicht anders sehen) nicht denkbar ohne substantielle materielle Verbesserungen auch im Lebensniveau sowohl der einzelnen als auch der sozialen Gruppen und Nationen. In einem Komplex empirischer Entwicklungstheorien ist das durchaus zusammenzubringen mit Strategien gesteigerter Produktivität im Agrarbereich und/oder einer verbesserten Industrialisierung, mit Partizipationsgesichtspunkten, dem Performanz- und Distributionsvermögen eines Regierungssystems, ohne daß man daraus gleich ein durchgängig westliches Entwicklungsmuster in allen möglichen wirtschaftlichen, kulturellen, politischen Details folgern müßte. Ähnliches würde für jede praktisch einzuschlagende Entwicklungspolitik gelten, allerdings auch die Umkehrung, daß eine Entwicklungspolitik nur dann ihrem Namen gerecht wird, wenn sie die individuellen wie kollektiven Lebensbedingungen auch materiell verbessert.

Diese soeben referierten drei Erfahrungsniveaus im westlichen Vorbild sollen das Beziehungsgefüge systemischer wie individueller Entwicklung anzeigen. Was bedeutet all dies für die Konzeptualisierung und Planung politischer Entwicklung?

\section{II}

\section{Zum Problem einer angemessenen Konzeptualisierung und Planung politischer Entwicklung}

Daß der paradigmatische Rang der historischen Selbsterfahrung Europas und Nordamerikas auch die im engeren Sinne politische bzw. politikwissenschaftliche Entwicklungsdiskussion beherrscht, kann nicht deutlich genug betont werden ${ }^{35}$. Franz Nuscheler ${ }^{36}$ hat in diesem Sinne schon vor Jahren entschieden festgestellt: „Die Theorien über das Entstehen des modernen Staates, die meist einseitig am kontinentaleuropäischen und absolutistischen Vorbild orientiert sind, werden . . .

33 Abgehoben wird hier auf die sog. Princeton-Reihe „Studies in Political Development ${ }^{\alpha}$, bes. auf Band 7: Leonard Binder u. a. (Hgg.), Crises and Sequences in Political Development, Princeton 1971.

$34 \mathrm{Vgl}$. die entsprechende Kritik bei Mark Kesselmann, Order or Movement? The Literature of Political Development as Ideology, in: World Politics XXVI 1973, $139 \mathrm{ff}$.

$35 \mathrm{Vgl}$. statt vieler Huntington (zit. Anm. 9) sowie Nohlen/Nuscheler (zit. Anm. 1).

36 Nuscheler, Theorien zur politischen Entwicklung (zit. Anm. 1), 75. 
zu Aktionsmodellen für die Staatenbildung innerhalb der dekolonisierten Dritten Welt. Aus der vergleichbaren historischen Ausgangssituation des europäischen Feudalismus und der heutigen traditionalen Entwicklungsgesellschaften wird die politische Therapie abgeleitet." Man muß das noch um Nordamerika erweitern, welches ja den Verfassungsstaat der Neuzeit mitgeboren hat, und man sollte vielleicht noch die vergleichbare historische Situation in Parenthese setzen - dann hat man recht genau den vorherrschenden Argumentationsduktus der politischen Entwicklungstheorie. Man kann sozusagen beliebig und auf Verdacht von den Erscheinungsformen westlicher politischer Modernität ausgehen, um thematisch analoge und dann mit entsprechenden Zielsetzungen argumentierende Entwicklungstheorien für den politischen Bereich $\mathrm{zu}$ finden, und schon wird man sie haben ${ }^{37}$ :

- Der moderne westliche Staat zeichnet sich durch eine zentrale staatliche Autorität und den Aufbau einer ihr gefügigen staatlichen, bürokratisch aufgezogenen Verwaltung aus; entsprechend gibt es einen "administrative approach“ in der politisch-theoretischen Entwicklungsdiskussion.

- Der moderne westliche Staat kennt eine vergleichsweise hohe Identifikation des Staatsbürgers mit seinem jeweiligen Gemeinwesen. Entsprechend finden sich wichtige entwicklungstheoretische Beiträge zum Thema "nation building“ durch Integration.

- Im modernen westlichen Staat werden zahlreiche Entscheidungen getroffen auf der Grundlage ausgeweiteter Partizipation und gruppenspezifischer Interessenwahrnehmung. Eine entsprechende Rolle spielt die entwicklungstheoretische Diskussion um Partizipation, Mobilisierung des einzelnen, Interessenaggregierung durch Gruppen etc.

- Im modernen westlichen Staat tragen Schichtungsbilder und Klassengefüge erkennbar die staatlich-gesellschaftlich vertikalen und die binnengesellschaftlich horizontalen Konfliktlösungsmuster. Entsprechend gibt es eine Forschungsrichtung, die sich mit der Identifizierung und Größe entsprechender sozialstruktureller Variablen beschäftigt.

Das Ganze ist nahezu beliebig fortsetzbar. Es werden die wesentlichen politischen Erscheinungsformen des modernen westlichen Industriestaates als Maßstab gesetzt, und die überwiegend empirisch arbeitende Entwicklungsländerforschung hat es dann leicht, die politischen Systeme der Dritten Welt daraufhin durchzurechnen, in welchem Umfang und wann das Paradigma erreicht wird oder nicht. Auch dort übrigens, wo man stärker normativ ausgerichtet ist, wird man fremde Entwicklungspotentiale nach dem Korrespondenzverhältnis mit den eigenen Idealen durchsehen. Der nordatlantische Maßstab taucht manchmal in entwaffnender Naivität auf - so etwa, wenn in dem seinerzeit berühmt gewordenen MIT-Symposium über „The Emerging Nations ${ }^{38 \text { " }}$ zwar zunächst festgestellt wurde: „Das Problem politischer Entwicklung besteht ganz deutlich nicht darin, Kopien (replicas) westlicher Institutionen in all den Gesellschaften im Ubergang zu realisieren “, um dann aber sogleich zu der Forderung überzuleiten: „Es kommt vielmehr darauf an, funktionale Aquivalente der Essenz demokratischer Regierung und Politik zu

37 Genaue Literaturnachweise für die im folgenden Text gemachten Andeutungen bei Nuscheler, Heimer, Berg-Schlosser (zit. Anm. 1).

38 Max F. Millikan/Donald L. M. Blackmer (eds.), The Emerging Nations. Their Growth and United States Policy, Boston/Toronto 1961, 89 (zit. nach Nuscheler, Theorien zur politischen Entwicklung (zit. Anm. 1), 101. 
verwirklichen." Weniger leicht zu durchschauen, aber dafür um so häufiger, findet sich das westliche Vorbild in der etwas sublimeren Verpackung zunächst abstrakt vorgetragener Modelle, z. B. in der entwicklungstheoretisch ausgerichteten Systemanalyse Gabriel Almonds ${ }^{39}$ und seiner Schule, die mit ihrem von Easton übernommenen Input-Output-Schema letztlich am staatstheoretisch-liberalistischen Dualismus von "Staat" und "Gesellschaft" bzw. an dem das Herrschaftssystem der Vereinigten Staaten kennzeichnenden Tauschcharakter des politischen Prozesses festhält.

Der nordatlantische Maßstab ist nicht nur eine Prärogative sogenannter bürgerlicher Analysen. Wenn etwa Breuer - Hartmann - Lederer ${ }^{40}$ im einleitenden Kapitel ihres Buches „Revolution in Lateinamerika“ behaupten: „Mit der kubanischen Revolution ist der Sozialismus als Gesellschaftsordnung auch in Lateinamerika aus der Welt der Vorstellung in die Wirklichkeit getreten", dann haben wir es mit nichts anderem zu tun als mit einer marxistischen Variante des westlichen Selbsterfahrungsprozesses. Ähnlich heißt es in einer neueren „Einführung in die politische OKkonomie des Sozialismus" eines Ost-Berliner Autorenkollektivs"1: "Die dritte Etappe der Entwicklung des Sozialismus beginnt 1959/60 und wird charakterisiert durch den Triumph des Sozialismus in einer großen Gruppe von Ländern, durch das starke Anwachsen der Kräfte, die für den Sieg des Sozialismus in der ganzen Welt kämpfen ..." Ist Sozialismus hier wirklich mehr als das System-Leitbild des europäischen Staatssozialismus ${ }^{42}$, der sich ja in der Vergangenheit schwer genug daran getan hat, mit den chinesischen oder kubanischen oder gar afrikanischen „Abweichungen“ fertig zu werden? Etwas allgemeiner und zugleich konkreter gesagt: Wenn man sich nicht scheut, das eigene dichotome Klassenmodell auf Gesellschaften mit überwiegender Marginalität und Subsistenzwirtschaft zu übertragen oder wenn „der Feudalismus-Begriff für alles herhalten muß, was nicht eindeutig als kapitalistisch zu bestimmen ist", dann bietet man nicht mehr an als eine marxistische Variante der oft gescholtenen westeuropäisch-nordamerikanischen Modernisierungsmethoden ${ }^{43}$.

Was insgesamt vielleicht noch schwerer wiegt: Auch die Dritte Welt selbst verschleiert nur mühsam - wenn überhaupt - ihre Annahme des westlichen Vorbilds. Wo z. B. die Entwicklungsdiskussion der Lateinamerikaner stark ist - etwas davon streifte ich schon eingangs - ist sie entweder eine entwicklungspolitische Spielform der europäisch-marxistischen Imperialismus-Theorie oder eine Fortsetzung der im „bürgerlichen“ Westen seit Jahren angestellten Überlegungen zu den von konkreten Elitenpotentialen her zu realisierenden Alternativen Nationalkapitalismus, Staatskapitalismus, Entwicklungssozialismus ${ }^{44}$ bzw. eine für die eigene Region aktualisierte „europäische“ Integrationsdebatte ${ }^{45}$.

Man darf feststellen: Auch die politologischen Entwicklungstheorien und -analysen sind bei näherem Hinsehen geschrieben unter der Annahme der Vorbildlichkeit des nordatlantischen Raumes, wobei die verschiedenen Schulen und Lager eher Präfe-

$39 \mathrm{Vgl}$. die entsprechende Ideologie-Kritik bei Mols (zit. Anm. 31), 101, 128.

40 Wilhelm M. Breuer/Bernd Hartmann/Herbert Lederer, Revolution in Lateinamerika, Köln $1969,1$.

41 Autorenkollektiv, Einführung in die politische Okonomie des Sozialismus, Frankfurt 1974, 35 f.

$42 \mathrm{Vgl}$. Nuscheler (zit. Anm. 5), 199

43 Ebd.

44 Vgl. Helio Jaguaribe, Political Development: A General Theory and a Latin American Case Study, New York u. a. 1973 , bes. Kap. 15.

45 Für Lateinamerika vgl. z. B.: Ronald Hilton, The Movement toward Latin American Unity, New York u. a. 1969; Banco Interamericano de Desarrollo, Factores para la integración Latinoamericana, México 1969 (1. Aufl. 1966); Helio Jaguaribe u. a. (eds.), La Dependencia Político-Económica de América Latina, México 1969, dort bes. das sog. MADIAL-Modell. 
renzen innerhalb des gegebenen Anschauungssubstrates bzw. seiner mutmaßlichen Extrapolationen denn eigentliche Alternativen darstellen. Das meiste, was über das Thema politische Entwicklung in Europa und Nordamerika vorgelegt wurde, wird man zudem gleichfalls als politische Modernisierungstheorien relativieren müssen, die nach dem Prinzip der Defizit-Feststellung am in der Dritten Welt ausgesuchten Untersuchungsfeld operieren.

Daß somit die politischen Entwicklungstheorien die Entwicklungsnormen der allgemeineren Entwicklungsdiskussion reflektieren, kann natürlich nicht erstaunen. Denn zum einen partizipieren ja die Politologen mit den Ökonomen und den Historikern und den Soziologen an der gleichen Hermeneutik der sozialen Lebenswelt, hier also an der gleichen Erfahrung von vollzogener Entwicklung in Relation zu den traditionellen Gesellschaften. Zum anderen setzte eine speziell auf das Politische gerichtete Entwicklungsforschung ein gutes Jahrzehnt später ein als die allgemeine Entwicklungsdiskussion, so daß man sich vermutlich einer vorherrschenden Argumentationsführung gar nicht entziehen konnte.

Immerhin bleiben damit aber noch zwei gewichtige Fragen offen, die ich im restlichen Teil dieser Abhandlung wenigstens andeutungsweise beantworten möchte. Die erste Frage heißt: Wiederholen sich auch in der politischen Entwicklungsforschung jene für die allgemeineren Entwicklungsüberlegungen konstatierten QuasiAporien des westlichen. Vorbilds, daß man nämlich allem Fortschrittsoptimismus zum Trotz die historisch begrenzte Wiederholbarkeit und die nicht unbedingt zu wünschende Reproduktion des westlich-industriestaatlichen Leitbilds einerseits, andererseits eine Serie von vermittelten Lernerfahrungen $(=$ die in Abschnitt I genannten drei positiven Erfahrungsniveaus) auch hier bemerken muß? Und teilweise schon darin eingeschlossen -: Was wäre dann die Besonderheit der politikwissenschaftlichen Entwicklungsdiskussion im Verhältnis zu ihrem genus proximum?

Die historisch begrenzte Wiederholbarkeit des westlichen Vorbilds wird durchaus gesehen, wenngleich dieses manchmal etwas verklausuliert ausgesprochen wird, z. B. wenn man in der Parteienforschung zur Dritten Welt ${ }^{46}$ sich mit den Möglichkeiten einer Einparteiendemokratie anzufreunden sucht (freilich nicht ohne deren transitorischen Charakter hervorzuheben) oder wenn man in der vergleichenden Partizipationsforschung ${ }^{47}$ geringere Partizipationsvolumina in Entwicklungsländern damit zu entschuldigen sucht, daß man auf die Lähmungen des politischen Entscheidungsprozesses für den Fall einer sehr ausgeweiteten Partizipation hinweist. Ein anderes, in Abschnitt I bereits angedeutetes Beispiel: Auch Huntingtons $^{48}$ „leviathanischer" Primat politischer Ordnung oder das kaum hinterfragte Elitenkonzept der Almond-Binder-Schule ${ }^{49}$ dürfte als ein indirektes Eingeständnis ungewisser Imitationschancen anzusehen sein. Die historisch begrenzte Wiederholbarkeit des westlichen Vorbilds wird auch dort angesprochen, wo man die Möglichkeiten eines eigenen Entwicklungssozialismus überdenkt $t^{50}$, wobei sich hier gelegentlich die Skepsis hinsichtlich einer Nachahmungswürdigkeit des russischen Vor-

\footnotetext{
46 Vgl. Mols (zit. Anm. 8), 216

47 Vgl. das Schlußkapitel bei Gabriel A. Almond/Sidney Verba, The Civic Culture. Political Attitudes and Democracy in Five Nations, Boston and Toronto 1965.

48 Vgl. bes. dessen Buch: Political Order in Changing Societies, New Haven and London 1968.

49 Wie es außer in der Princeton-Reihe (vgl. Anm. 33) auch greifbar ist in: Gabriel A. Almond/Scott C. Flanagan/Robert J. Mundt (eds.), Crisis, Choice, and Change, Boston 1973.

50 Vgl. die Anm. 44 zit. Arbeit von Jaguaribe.
} 
bildes mit einer gleichen Skepsis hinsichtlich seiner Nachahmungsmöglichkeiten mischen kann ${ }^{51}$ (dies gilt allerdings nicht für den ungleich "gesellschaftlicheren" Sozialismus Jugoslawiens, der durchaus Attraktion ausstrahlt).

Immerhin ist in der politologischen Literatur normalerweise die Frage nach der faktischen Wiederholbarkeit des westlichen Vorbildes deutlicher verneint worden als die nach der Wünschbarkeit, denn dies hätte eine Herrschaftskritik spätindustrieller Gesellschaften vorausgesetzt, wie es sie breiter erst in jüngerer Zeit in Form der Krisenforschung ${ }^{52}$ gibt (oft am fruchtbarsten am Rande der akademischen Diskussion ${ }^{53}$ ).

Ungleich forscher dagegen - um dies hier einzuschieben - hat man sich in dieser Hinsicht seitens herausragender Führungspersönlichkeiten und/oder Bewegungen in der Dritten Welt immer dann geäußert, wenn man das Programm eines eigenen Weges verkündete, etwa als ein Drittes zwischen Kapitalismus und Sozialismus oder als ein eigener, nicht-westlicher Entwicklungssozialismus. Die Entschiedenheit, mit der man sich seinerzeit im justicialismo Peróns $s^{54}$ oder im camino chileno der Unidad Popular ${ }^{55}$ oder im Etat Pilote des Sékou Touré5 oder im „wissenschaftlichen Sozialismus" des nasseristischen Ägypten eigener Transformationsziele versichert hat, stand freilich regelmäßig in einem sichtlichen Gegensatz zur konstruktiven Konkretheit dessen, was man längerfristig durchführte. Gute Kenner des afrikanischen Sozialismus ${ }^{57}$ urteilen in fast übereinstimmenden Formulierungen über die unübersehbare Kluft zwischen „ideological prescriptions“ und „policy impact".

Zurück zur wissenschaftlichen Diskussion! Wie steht es unter politikwissenschaftlichen Gesichtspunkten mit den drei Erfahrungen, die das westliche Vorbild der Entwicklungsdiskussion insgesamt zur Verfügung stellt?

Die erste Erfahrung hatte gelautet: Unsere Sensibilisierung für den Unterschied zwischen Modernisierung und Entwicklung weist ersterer den Rang eines aus konkreten Einzelmaßnahmen notwendigen take-off zu. Davon verspreche ich mir für die politikwissenschaftlichen Modernisierungstheorien mittlerer Reichweite ungleich weniger als von dem, was die anderen Sozialwissenschaftler beizutragen vermögen. Es hängt dies mit dem Spezificum politikwissenschaftlicher Entwicklungsüberlegungen zusammen: Bei näherem Hinsehen zeigt sich nämlich ein gewisser Bruch zwischen der allgemeinen neueren Entwicklungsdiskussion und den Konzeptualisierungen politischer Entwicklung im engeren Sinne. Beide Klassen von Ansätzen, die allgemeine Klasse und die Subklasse „politische Entwicklung“, sind zwar im wesentlichen Theorien partieller Modernisierung auf der Basis historischer Erfahrung (im Westen). Aber da politische Entwicklung im Gegensatz zu anderen Entwicklungsmaterien viel weniger betrieben werden kann ohne deutliche

51 Ebd. 242.

52 Vgl. Martin Jänicke (Hg.), Herrschaft und Krise. Beiträge zur politikwissenschaftlichen Krisenforschung Opladen 1973; ders. (Hg.), Politische Systemkrisen, Köln 1973 (dort bes. Jänickes Einleitungskapitel S. $14 \mathrm{ff}$.).

53 Das Anm. 16 zit. ZEIT-Buch ist hier ein überzeugendes Beispiel.

54 Vgl. Peter Waldmann, Der Peronismus 1943-1955, Hamburg 1974.

55 Vgl. Alejandro Foxley (ed.), chile: búsqueda de un nuevo socialismo, Santiago de Chile 1971. Interessant in unserem Zusammenhang bes. der daselbst von Jacques Chonchol Ch. gelieferte Beitrag "Elementos para una discusión sobre el camino chileno hacia el Socialismo".

56 Vgl. Reinhard Kapferer, Sozialismus in Afrika - Agypten und Guinea. Zwei Fallstudien zum ideologischen Selbstverständnis und zur politischen Soziologie unterentwickelter Gesellschaften, in: aus politik und zeitgeschichte vom 17 . Juni 1972.

$57 \mathrm{Vgl}$. Anderson/von der Mehden/Young (zit. Anm. 7), 10; Marion Mushkat, der afrikanische Sozialismus, in: PVS XII/2 1971, $220 \mathrm{ff}$. Auch Mushkat spricht von einer ${ }_{n}$ tiefen Kluft, die die Programme von der Realität trennt“ (250). 
ordnungspolitische Leitvorstellungen in Anlehnung an vorhandene historische Potentiale, besitzen die in den Theorien zum Ausdruck gelangenden Ratschläge eine unterschiedliche Verbindlichkeit. Wirtschaftliche oder demographische Entwicklungstheorien lassen sich häufig umsetzen in technologische Verbesserungsvorschläge von ubiquitärer Gültigkeit. $\mathrm{Da}$ eine Steigerung materieller Úberlebenschancen bzw. eine Verbesserung materieller Wohlfahrt nicht ohne eine bestimmte Relation von Wirtschaftswachstum und Bevölkerungszunahme vor sich gehen kann, auch nicht ohne Reinvestitionsraten, für deren notwendige Höhe man auf bisherige Erfahrungen in der entwickelten Welt zurückgreifen kann, liegt auf der Hand. Die Einführung jedoch von neuen politischen Strukturen wie Planungsmechanismen oder politischen Parteien oder von Institutionen öffentlicher Kommunikation und Kontrolle der Regierenden setzen viel mehr an ordnungspolitischen Leitbildern und Globalentwürfen voraus (bzw. zeitigen weitreichendere ordnungspolitische Konsequenzen), die dann, wenn man die Reproduzierbarkeit des westlichen Vorbilds gar nicht mehr für wünschbar hält, ungleich intensiver gedanklich vorbereitet und erdiskutiert werden müssen. Man kann das auch anders formulieren und sagen: Wollte man Komponenten im westlichen Vorbild unterscheiden, dann würde ein Ökonom viel selbstgewisser auftreten (können) als ein Politologe. Oder in der Frage von Charles Lewis Taylor ${ }^{58}$ : „Letztlich sind sich alle darin einig, daß die Förderung des Wohlstands, oder zumindest die Erhöhung der allgemeinen Wohlfahrt, gut ist, und daß dies das eigentliche Ziel ökonomischer Entwicklung ist. Was ist jedoch das Ziel politischer Entwicklung?" Denn, so schreibt er weiter, es gibt „keine sichtbare politische Ware. Nichts bildet die genaue Analogie zu Wohlstand. Politische Entwicklung ist nicht die Anhäufung von immer mehr Macht, Autorität und Souveränität ... D Da nun unverhältnismäßig viel, was bisher von den Politologen vorgelegt wurde, im Rahmen der fact finding mission des behavioristischen Ansatzes erarbeitet wurde, eignet es sich ungleich sicherer zur schon bemerkten Defizitmessung als zu praktischen politischen Maßnahmen oder gar zu ordnungspolitischen Alternativentwürfen. Vielleicht etwas überzogen: Während die Beiträge der Ökonomen und Soziologen oft gerade wegen ihrer mehr technologischen Natur und/oder wegen ihres Aufgreifens unbestrittener Wohlfahrtsnormen eine relativ unproblematische Umsetzung in eine entsprechende Entwicklungstechnologie ermöglichen, ist eine analoge technologische Ausnutzung politologischer Entwicklungserkenntnisse fragwürdig. Der spätaufklärerische Fortschrittsoptimismus hält sich zwar, soweit ich sehe, auch bei vielen Politologen, aber dort, wo es um praktische politische Entwicklungspolitik geht, sinkt vieles zur Unverbindlichkeit einer interessanten Deskription herab. So daß man sich überhaupt fragen sollte, ob Theorien, die auf positivistischen Fakten-Analysen aufbauen und dann nicht darüber hinauskommen, überhaupt zur Vorstellung ordnungspolitischer Entwürfe mit explizierter Rationalität fähig sind.

Die zweite Erfahrung kreiste um die Natur von Entwickeltsein, nenne man dies nun Entwicklungsdynamik oder Zielsetzungsautonomie oder mit Julius Nyerere self-reliance. Zielsetzungsautonomie ist eine gesamtgesellschaftliche Qualität in Relation zur konkreten historischen Umwelt, eben die Chance der aktiven Mitgestaltung, kybernetisch gesprochen parthenogenetisches Lernen. Zielsetzungsautonomie wird in einer bi- oder tripolaren Welt oder in einer Situation eines immer evidenter

58 Charles Lewis Taylor, Indikatoren politischer Entwicklung, in: Nohlen/Nuscheler (zit. Anm. 1), 280 f. 
werdenden Nord-Süd-Gefälles oder auch angesichts der großen internationalen Wirtschaftsverflechtungen sicher bis auf weiteres oder sogar für immer ein Ideal bleiben, aber doch ein Ideal, dem man sich in der Dritten Welt etwas mehr als bisher nähern kann. Hier würde ich nun die erkennbaren Einflüsse bzw. die künftigen Einflußchancen von Diskussionsbeiträgen zu politischer Entwicklung günstiger beurteilen als hinsichtlich der ersten Erfahrung.

Bekanntlich hat die neuere Entwicklungsdiskussion eine übermäßig lange Zeit darunter gelitten, daß das ökonomische Modernisierungsdefizit in der theoretischen Betrachtung geradezu monopolisiert wurde. Nicht nur, daß die Ókonomen das Sagen hatten, daß die „Ursachen der Armut in der Dritten Welt ${ }^{59}$ “ als das eigentliche Entwicklungsproblem angesehen wurden, und daß man bis in die unmittelbare Gegenwart hinein überwiegend sozioökonomische Indikatoren zur Messung von Entwicklungsniveaus verwendet, viele hervorragende Politologen und Soziologen waren sich auch darin einig, daß erst auf der Grundlage einer zufriedenstellenden sozioökonomischen „Ausstattung“ politische Entwicklung nach westlichem Vorbild möglich sei (womit sie übrigens, vielleicht unbeabsichtigt, eine Art „bürgerliche" Version der marxistischen Unterbau-Überbau-These vortrugen). Inzwischen wissen wir aus Erfahrung, daß die angenommene Sequenz nicht existiert. Man erkennt aber immer mehr, daß Entwicklung eine gesamtsystematische Transformationsaufgabe ist und daß folglich alle gesellschaftlichen Subsysteme, also das wirtschaftliche System, das kulturelle System, das soziale System im engeren Sinne und das politische System in Form permanenter Interaktionen an dieser Transformation beteiligt sein müssen. Wahrscheinlich ist nun, daß dabei der politischen Führung die Initiative und die permanente Steuerungsrolle zukommt, jedenfalls würde ich dies aus solchen Lektionen wie China, Ägypten, Kuba, Peru, Mexiko entnehmen, alles Länder, in denen Hervorragendes an gesamtgesellschaftlicher Transformation geleistet wurde. Jene relative Zielsetzungsautonomie, von der ich mehrfach sprach, ist schon wegen der weltwirtschaftlich strukturellen Bindungen moderner Volkswirtschaften am wenigsten im ökonomischen Bereich zu vergrößern und am ehesten noch durch eine entschlossene politische Führung, der es gelingt, alle übrigen sozioökonomisch-kulturellen Handlungsfelder $\mathrm{zu}$ aktivieren und auf eine gewisse Unabhängigkeit "nach außen“ zu achten, also externen Einflüssen autonomer zu begegnen (einschließlich der Reaktions-Aktionsfähigkeit in bezug auf extern vorgeschlagene/eingebrachte/prämierte Entwicklungstheorien/ Technologien etc.). Richard F. Behrendt ${ }^{60}$, der ja als einer der ersten Entwicklungsforscher mit dem Begriff der kulturellen Dynamik gearbeitet hat, nennt als Instrumentarien einer sozialen Entwicklungsstrategie: Planung, Fundamentaldemokratisierung, plurale Mobilität von Entwicklungsträgern, Nivellierung der Machtstruktur und globale Entwicklungsförderung. Das sind doch Dinge, die von einem politischen Entscheidungswillen angeregt und dann von ihm gesteuert und überwacht werden müssen, d. h. von Instanzen, die über genügende Machtressourcen verfügen, um sich durchsetzen zu können, wobei diese Instanzen angewiesen sind auf die Unterstützung von Eliten und Sub-Eliten, die die Notwendigkeit von gesamtsystemischen Transformationen begriffen haben. Um hier keine Miß-

59 So ist noch der 2. Hauptabschnitt bei Bohnet (zit. Anm. 1) überschrieben. Vgl. hier auch: Dietrich Kebschull, Entwicklungspolitik. Eine Einführung, Düsseldorf 1971; Bruno Fritsch (Hg.), Entwicklungsländer, Köln/Berlin 1968. 60 Zit. Anm. 28; ders., Die Zukunft der Entwicklungsländer als Problem des Spätmarxismus, in: Futurum 4,
1970, $574 \mathrm{ff}$. 
verständnisse zu erzeugen: Ich will nicht aus dem alten entwicklungsökonomischen Primat einen neuen Primat der Politologen machen, sondern den Stellenwert der politikwissenschaftlichen Diskussionsteilnahme auf der Basis von Úberlegungen aufzeigen, die den Rang des gouvernementalen Bereiches und zugeordneter gesellschaftlicher Unterstützungsleistungen im Zielsetzungsvermögen eines gesellschaftlichen Systems - eben auch in der Dritten Welt - erkennen lassen.

Noch etwas anderes kommt hinzu. Auf längere Sicht werden die Politologen der Dritten Welt gegenüber in die Rolle eines lästigen Mahners kommen, glaubt man dort auch oft genug, durch partielle Modernisierungen, vor allem auf den Gebieten der Wirtschaft, der infrastrukturellen Technik und vielleicht noch der Volksbildung entwickelt zu werden. In einer Vorschau auf die arabische Kultur des Jahres 2000 aus der Feder des ägyptischen Erziehungsministers Kamal ed-Din Husain ${ }^{61}$ sieht man sich befähigt, zum gegebenen Zeitpunkt der europäisch-amerikanischen Kultur als erfolgreiche Konkurrentin entgegenzutreten. Die wesentlichen Attribute dieser arabischen (!) Zukunft heißen: Stahlwerke, Atommeiler, Volksbibliotheken. Mit geringfügigen Modifikationen hätte ich auch von Brasilien oder Algerien sprechen können. Ein anderes Beispiel: Darcy Ribeiro ${ }^{62}$ erwartet in seinem Buch "Der zivilisatorische Prozeß" eine postindustrielle Einheitskultur auf der Grundlage einer "Thermonuklearen Revolution“. Auf die ihm von Heinz Rudolf Sonntag63 gestellte Frage nach den Trägergruppen dieses technologischen Durchbruchs kommt die reichlich kurzgreifende Antwort: „Der Protagonist einer technologischen Revolution ist die technologische Revolution, d. h. eine Reihe von vorteilhaften Neuerungen in der Weise, die Dinge zu machen. Das ist der kausale Faktor, der Protagonist, der Motor der Geschichte.“ Der Korrekturbeitrag einer politischen Entwicklungsdiskussion wird darin bestehen müssen, permanent und so konkret wie möglich an die Aktivitäten des politischen Systems zu erinnern. Denn weder kommt eine arabische Zukunft von selbst, noch machen sich die technischen Dinge selber. Auch hier geht es nicht um einen Primat des Politischen, sondern um seinen Beitrag.

Die Durchsicht der paradigmatischen Möglichkeiten des westlichen Vorbilds hatte noch eine dritte Erfahrung nahegelegt, nämlich die, in einer Art phänomenologischen Reduktion nach solchen Elementen zu suchen, die jenseits der zufälligen Ausprägung im historischen Erscheinungsbild zur Moderne überhaupt z.u gehören scheinen. Es ist dies der abstrakteste Erfahrungsrang des westlichen Vorbilds. Gemeint sind einige Topoi, die in der neueren Entwicklungsdiskussion auch interdisziplinär immer mehr in den Vordergrund treten und die die Erfahrung des Westens so repräsentieren sollen, daß man nicht von vornherein zur Imitation der großen gesellschaftspolitischen Ordnungsformen der Ersten und Zweiten Welt verpflichten will.

Solche Elemente sind z. B.: Gleichheit, Differenzierung, Kapazität, Rationalität, Lebensqualität, Innovation, Kritik . . ${ }^{64}$ Übersetzt in etwas mehr Anschauung:

- Wie immer die konkrete Ausgestaltung gesellschaftlicher Veränderung hier im Westen aussieht - das Streben nach und das Erreichen von mehr gesellschaftlicher und schließlich auch politischer Gleichheit gehören dazu.

61 Uwe Simson, Typische ideologische Reaktionen arabischer Intellektueller auf das Entwicklungsgefälle, in: KZS, Sonderheft 13: Aspelite der Entwicklungssoziologie, 1969, $136 \mathrm{ff}$., bes. 145.

62 Darcy Ribeiro, Der zivilisatorische Prozeß, Frankfurt 1971 (bras. Orig. 1968).

63 Ebd. 278 (Aus dem als Anhang aufgeführten Gespräch zwischen Ribeiro und Sonntag).

64 Ein Teil dieser Elemente findet sich in dem in Anm. 33 zit. Krisenband von Binder et al. Vgl. auch Bruno Fritsch, Die Vierte Welt. Modell einer neuen Wirklichkeit, Stuttgart 1970. 
- Wie immer unterschiedlich die konkreten historischen ökonomischen und politischen Systeme in den Ländern des nördlichen Atlantik sich entwickelt haben mögen - die durchgehende Ausweitung ihrer Leistungs- und Planungskapazitäten ebenso wie die zunehmende Differenzierung der politisch-ökonomisch-sozialen Arbeitsteilung gehören dazu.

- Wie immer unterschiedlich groß und manchmal auch problematisch die Absage an geistige/soziokulturelle/politische etc. Traditionen bei uns gewesen sein mag - der Erwerb gesteigerter Rationalität als Kalkül des Grenznutzens in Zweck-Mittel-Relationen gehört dazu.

- Und umgekehrt: Wie immer auch die entwickelte Welt an sehr vielen eingespielten politischen/gesellschaftlichen/ökonomischen Problemlösungsmustern schon deshalb festhalten muß, weil keine Gesellschaft zu vertretbaren Kosten ihre Routineangelegenheiten nur durch Experimente erledigen kann - Innovation und Kritik gehören zu ihr, weil ausschließlich Routine-Reaktionen auf neue Situationen national wie international zum politisch-gesellschaftlichen Kollaps führen.

- Wie immer unterschiedlich man in der Bundesrepublik Deutschland und in Rußland und in Schweden und in den USA Lebensqualität definieren mag ein relativ hohes Niveau auch materieller Lebensbedingungen gehört dazu.

Ein Geistesgeschichtler könnte hier mühelos viele der großen Themen der europäischen Soziologie wiederentdecken, aber das Entscheidendere ist doch, daß dies Grunderfahrungen für die Entwicklungsdiskussion sind, die, versehen mit einem Minimum an ideologischer Vorbelastung, sowohl bei uns eine breitere interdisziplinäre wie politische Verständigung als auch ein verhältnismäßig wenig gängelndes Gespräch mit der Dritten Welt erlauben, deren immer wieder aufkommender Versuch, die eigene Kultur und Tradition unter betonter Absetzung vom Westen zu beleben, um auch auf diese Weise sich selbst zu entwickeln (Revivalismus - Nativismus ${ }^{65}$ ), hier ein modernes, kontrasthaftes Pendant erhielte. Für die politische Entwicklungsdiskussion ergibt sich daraus eine Reihe von Konsequenzen. Zum Beispiel:

- nach Entwicklungsmodellen und nach einer praktischen Entwicklungspolitik zu suchen, die einigermaßen gleichmäßig interaktiv gesicherte Ausweitungen von Gleichheit, Differenzierung, Kapazität, Rationalität, Lebensqualität usw. ermöglichen;

- den genauen Stellenwert politischer Entwicklungsleistungen innerhalb der gesamtgesellschaftlichen Transformationsanstrengungen $\mathrm{zu}$ bestimmen, sich also präzise zu überlegen, was der konkrete Anteil des politischen Systems auf allen Ebenen des Entwicklungssyndroms bedeutet;

- nicht nach der schlechthin gerechten und guten Gesellschaft zu suchen, nach einem neuen Menschen auf einer neuen Erde, sondern nach einer Gesellschaft oder nach Staaten, die deutliche Steigerungsdifferentiale in bezug auf ein Mehr an Gleichheit, ein Mehr an Innovation, ein Mehr an materieller Lebensqualität usw. aufweisen.

Wenn ich das recht sehe, zeigt im Augenblick die Arbeit mit dem Entwicklungssyndrom die modernste Richtung innerhalb der politischen Entwicklungsländer-

65 Vgl. Bryce Ryan, Die Bedeutung der Revitalisationsbewegungen für den sozialen Wandel in den Entwicklungsländern, in: KZS, Sonderheft 13, Aspekte der Entwicklungssoziologie, 1969, $37 \mathrm{ff}$. 
forschung an. Für die Vereinigten Staaten würde ich auf Leonard Binders ${ }^{66}$ „Crises and Sequences in Political Development" verweisen (ich meine ausdrücklich den analytischen Ansatz als solchen, nicht die mitgelieferten Interpretationen!); für Deutschland u. a. auf Peter Waldmanns67 Untersuchung über den Peronismus. Die Logik des Vorgehens läßt sich dabei leicht skizzieren: Während die traditionelle Entwicklungsländerforschung nach korrekten Wahlen und funktionierenden Parteien und Verbänden fragte, würde man hier schlicht auf die Ausweitung, Stagnation oder Verminderung des Partizipationsvolumens und auf Sprünge in der Partizipationsqualität achten. Während die traditionelle Entwicklungsländerforschung auf Demokratisierungsvorstellungen nach angelsächsischem Vorbild fixiert war, würde man nun stärker auf ein Entsprechungsverhältnis von Gleichheit, Kapazität und Lebensqualität sehen, z. B. auf ein Entsprechungsverhältnis von Partizipation und Distribution, Partizipation und Penetration, Penetration und Distribution etc. Gerade bei dem letzten Beispiel liegt es auf der Hand, daß diese Art von empirischer Entwicklungsforschung grundsätzlich fähig ist, die bisherigen Einwände kritisch-dialektisch-politökonomischer Provenienz gegen die sogenannte formale Demokratie des Westens, die ja auch von sehr vielen Menschen in der Dritten Welt geteilt werden, ernst zu nehmen und entsprechend zu berücksichtigen.

Ich sagte „empirische Entwicklungsforschung“. Die Arbeit mit dem Entwicklungssyndrom erlaubt es, empirisch auf der Basis normativ-historischer Grundwerte zu arbeiten, wissenschaftlich und politisch. Und diese Grundwerte sind nichts anderes als solche Erfahrungen im westlichen Vorbild, die bei allem Gewicht nicht zur Úbernahme klischeegleicher westlicher Einstellungen und Strukturen verpflichten.

Noch eine Schlußbemerkung: Ich habe in dieser kurzen Abhandlung bewußt auf eine geschlossene Definition von politischer Entwicklung oder gar auf die Konstruktion eines politischen Entwicklungsmodells verzichtet. Es ging mir um ein paar grundsätzliche Bemerkungen zu einer von einem bestimmten historischen Anschauungssubstrat geprägten Diskussion.

Ich bin nicht Spezialist genug, um die Avancen und die konkreten Defizite der neueren Entwicklungsländerforschung im Detail zu übersehen, aber ich weiß aus einer geringen lateinamerikanischen Erfahrung, daß es nun, nach rund 25 Jahren wissenschaftlicher und politischer Befaßtheit mit diesen Dingen, gebotener ist denn je, solche Probleme mit den Kollegen und Politikern aus der Dritten Welt ungleich intensiver als bisher gemeinsam zu diskutieren, und ich habe mich hier um den Nachweis bemüht, wie wir unsere Erfahrungen in die Diskussion einbringen können, ohne aufdringlich zu sein. Auch das gehört zum Thema der Möglichkeiten und Grenzen des westlichen Vorbilds - vielleicht besonders für uns Politologen, die wir in der Vergangenheit wohl zu oft die Vorbildlichkeit des Verfassungsstaats der Neuzeit oder seiner sozialistischen Varianten überzeichnet haben.

66 Zit. Anm. 33.

67 Zit. Anm. 54. Ich selbst habe in dem in Anm. 8 zit. Aufsatz nach einem recht ähnlichen Ansatz die Transformationsleistungen der mexikanischen Revolutionspartei untersucht. 
The Problem of Western Pattern in the Latest Discussion on Political Development

\section{By Manfred Mols}

Current theories of development suffer from a cultural bias: that western society a western performance of the economy, western styles of life etc. are the final ends of development. Looked at more closely one realizes that the western paradigm reveals some ambiguities. To a certain extent a complete reproduction of the West ist neither tolerable nor realizable. On the other hand, the western paradigm provides a series of experiences which deserve attention. First: students of development have been made sensitive for a difference between development and modernization, representing the latter a sort of take-off quality for development. Second: Development must be defined as the relative autonomy of goalsetting of a societal system, driven by what some people have called cultural dynamics. Third: The very core of the western developmental paradigm cannot consist of ideals in form of structural analogies, but of a set of qualities like capacity, differentiation, quality of life etc., which are abstract enough not to call for direct imitation in a sense of structural correspondence, qualities which would nevertheless direct the attention to intrinsic features of the western experience.

Given this body of general considerations with regard to his subject, the author tries in a second part of his essay to apply this to political development. Especially the three experiences are repeated referring now to what the developmental theories of political scientists might contribute. One of the by-products of this second part is a delineation of developmental thinking as well as its practical impacts both in economics and in political science.

\section{The New Constitution of the People's Republic of China}

\section{By Oskar Weggel}

While the persons whom the Fourth National People's Congress has placed in the top positions of the People's Republic of China's administration, are more or less on the "moderate" side, explicit concessions have been made, in the text of the Constitution, to the cultural revolutionary leftists. One of them was even granted the privilege of explaining the changes made in the revised text of the Constitution to the plenum of the National People's Congress.

Unlike its predecessor of 1954, the revised Constitution is not the basic law for a "new democracy" but for a "socialist" state, with all the consequences resulting from this change. One of those consequences is e. g. the fact that only two socialist - kinds of ownership of the means of production are officially recognized now.

The revised Constitution very much resembles the "cultural revolutionary draft" of 1970 but the present authors have eliminated all reminiscences of Lin Piao and all reference to persons, including the names of Mao Tse-tung and Lin Piao.

The text of the 1975 Constitution is much more "transparent" than its predecessor of 19 years ago. The item of foremost importance now is the "Mao Tse-tung 